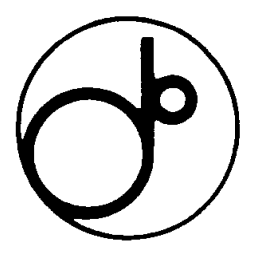

KEK Preprint 93-127

HUPD.9324

OUHEP 93-1

October 1993

$\mathbf{H}$

Beam Test of the SDC Double-sided Silicon Strip Detector

Y. Unno, F. Hinode, T. Akagi, T. Kohriki, N. Ujiie, KEK; Y. Iwata,

T. Ohmoto, T. Ohsugi, T. Ohyama, Hiroshima University; T. Hatakenaka,

N. Tamura, Okayama University; S. Kobayashi, A. Murakami, M. Tezuka, Saga University; R. Takashima, Kyoto Education University; T. Aso, H. Miyata, Niigata University; M. Daigo, Wakayama Medical College;

M. Higuchi, Tohoku-Gakuin University; I. Kipnis, H. Spieler, LBL;

J. DeWitt, D. Dorfan, A. Grillo, B. Hubbard, J. Rahn, W. Rowe,

H. Sadrozinski, A. Seiden, E. Spencer, A. Webster, M. Wilder, UC Santa

Cruz; M. Frautschi, J. Matthews, University of New Mexico; D. Kaplan, University of Oklahoma

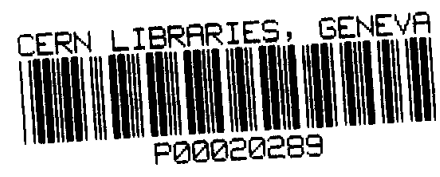

Presented at the IEEE 1993 Nuclear Science Symposium, Nov. 2-6, 1993, San Francisco, CA, U.S.A. 
National Laboratory for High Energy Physics, 1993

KEK Reports are available from:

Technical Information \& Library

National Laboratory for High Energy Physics

1-1 Oho, Tsukuba-shi

Ibaraki-ken, 305

JAPAN

Phone: $\quad 0298-64-1171$

Telex: $\quad 3652-534$ (Domestic)

(0)3652-534 (International)

Fax: $\quad$ 0298-64-4604

Cable: KEK OHO

E-mail: LIBRARY@JPNKEKVX (Bitnet Address) library@kekvax.kek.jp (Internet Address) 


\section{Beam Test of the SDC Double-sided Silicon Strip Detector}

Y. Unno, F. Hinode, T. Akagi, T. Kohriki, N. Ujiie, KEK; Y. Iwata, T. Ohmoto, T. Ohsugi, T. Ohyama, Hiroshima University; T. Hatakenaka, N. Tamura, Okayama University; S. Kobayashi, A. Murakami, M. Tezuka, Saga University; R. Takashima, Kyoto Education University; T. Aso, H. Miyata, Niigata University; M. Daigo, Wakayama Medical College; M. Higuchi, Tohoku-Gakuin University; I. Kipnis, H. Spieler, LBL; J. DeWitt, D. Dorfan, A. Grillo, B. Hubbard, J. Rahn, W. Rowe, H. Sadrozinski, A. Seiden, E. Spencer, A. Webster, M. Wilder, UC Santa Cruz; M. Frautschi, J. Matthews, University of New Mexico; D. Kaplan, University of Oklahoma

\section{Abstract}

A beam test was executed to evaluate the behavior of the first prototype radiation-hard double-sided silicon microstrip sensor for the SDC silicon tracking system. Pions of 4 $\mathrm{GeV} / \mathrm{c}$ in a test beamline at KEK illuminated three planes of detectors. The signals werc amplified, shaped, and discriminated with TEKZ bipolar analog LSI's, and the on-off levels were sampled at $10 \mathrm{MHz}$ clock with CMOS digital LSI's, asynchronously with beam triggers. The detectors were rotated in null and 1.0 Tesla magnetic fields. The efficiencies were found to be $98 \sim 99 \%$. The position resolutions were $12.5 \mu \mathrm{m}$, where the multi-strip hit fraction was $30-40 \%$. There was no essential difference in the performance of the $p$ and the n-sides. The multi-strip hit fraction showed a clear rotation and magnetic-field dependence. From the angles where the fractions were minimum in the $1 \mathrm{~T}$ magnetic field, the Hall mobilities of the electrons and holes were obtained to be $1391 \pm 43$ (clcctrons) and $325 \pm 30$ (holes) $\mathrm{cm}^{2} / \mathrm{Vs}$.

\section{INTRODUCTION}

A beam tcst (T280) was executed at a test beam line at KEK to evaluate the performance of the first prototype of a radiation-hard design of the double-sided silicon microstrip sensor (DSSS). The sensor was designed for the barrel section of the silicon tracking system of the SDC detector [1] for physics at the SSC $[2,3]$. Due to the high interaction rate, one of the most stringent requirements for the sensors is radiation hardness. After a number of studies on radiation effects, we had worked out a radiation-hard design and produced the first prototype of the DSSS, the specifications of which are summarized in Table I. Axial strips were processed at the $\mathrm{n}$-side and stereo strips (10 mrad stereo-angle) at the p-side, with a strip-pitch of $50 \mu \mathrm{m}$. It had AC coupling aluminum electrodes over the $\mathrm{p}$ - and $\mathrm{n}$-strips sandwiching a $\mathrm{SiO}_{2}$ insulator.

The SDC silicon tracking detector adopts a binary (on/off) readout scheme for its 6 million total readout channels. The shaping time constant of the front-end amplifier is expected to be about 20 ns to identify a hit to its own beam crossing, which occurs at a rate of $60 \mathrm{MHz}$. The silicon tracking system is imbedded in a solenoidal magnetic field of 2 Tesla for the momentum measurcment. In the layout of the silicon tracking system, charged tracks hit the sensors at a maximum angle of $14^{\circ}$ perpendicular to the axial strips and $60^{\circ}$ along the strips.

TABLE I

SPECIFICATIONS OF THE RADIATION-HARD SDC DOUBLE-SIDED SILICON STRIP SENSOR (BARREL)

(1) Substrate

Type n-type Si

Resistivity $\quad 4 \sim 8 \mathrm{k} \Omega \cdot \mathrm{cm}$

Thickness $\quad 300 \pm 10 \mu \mathrm{m}$

(2) Size

Overall dimension ${ }^{\mathrm{a}} \quad 60 \mathrm{~mm} \times 34.1 \mathrm{~mm}$

Stereo-effective area $\quad 58.8 \mathrm{~mm} \times 31.4 \mathrm{~mm}$

(3) Strip

Pitch $\quad 50 \mu \mathrm{m}$

Stereo angle $\quad 10 \mathrm{mrad}$

Implant width $\quad \geq 10, \leq 12 \mu \mathrm{m}$

AC electrode width $\quad<10 \mu \mathrm{m}$

Strip isolation of $n$-side $\quad \mathrm{p}^{+}$isolation line

(4) Capacitance

AC coupling $\quad \geq 20 \mathrm{pF} / \mathrm{cm}$

p-side (body+interstrip) $\quad \leq 1.2 \mathrm{pF} / \mathrm{cm}$

$\mathrm{n}$-side (body+interstrip) $\quad \leq 1.4 \mathrm{pF} / \mathrm{cm}$

(5) Bias resistor

Polycrystalline silicon $\quad 250 \pm 50 \mathrm{k} \Omega$

actual dimension is $60 \mu \mathrm{m}$ narrower due to sawing

\section{EXPERIMENT}

\section{Beamline Setup}

The experiment was set up at the $\pi 2$ test beamline of the 12-GeV proton synchrotron at $\mathrm{KEK}$ (Fig. 1). Five scintillation counters $\left(S_{1}, S_{2}, S_{3}, F_{1}\right.$, and $\left.F_{2}\right)$ were set to make a trigger for the data-acquisition sequence. $S_{1}, S_{2}$, and $S_{3}$ had a scintillator of $2 \mathrm{~cm}$ (width) $\times 2 \mathrm{~cm}$ (height) $\times 5 \mathrm{~mm}$ (thickness); $S_{2}$ and $S_{3}$ were placed close-by, but displaced to make an overlap of $1 \mathrm{~cm}$ in the horizontal direction; two small counters $\left(F_{1}\right.$ and $F_{2}$ ), each of which had dimensions of $5 \mathrm{~mm}$ $\times 1 \mathrm{~cm} \times 3.2 \mathrm{~mm}$, were placed in the shielding box for the detectors. The total coincidence of the five counters made a beam spot size of $5 \mathrm{~mm}$ (in horizontal) $\times 1 \mathrm{~cm}$ (in vertical) at the detectors. The timing was determined by $S_{3}$. Two 
Cerenkov counters were used to differentiate pions and electrons (the fraction of electrons was $-6 \%$ of the total particles). The coordinate system was right-handed and $+z$ in the beam-flow direction and $+y$ in the vertically upward direction.

A dipole magnel (USHIWAKA) with an opening aperture of $82 \mathrm{~cm} \times 40 \mathrm{~cm} \times 70 \mathrm{~cm}$ was placed in the down-stream of $S_{3}$ to provide a magnetic field ( $-y$ direction) having a maximum of 1.2 Tesla. An aluminum shielding box $(60 \mathrm{~cm} \times 39 \mathrm{~cm} \times$ $65 \mathrm{~cm}$ ) was held inside the opening. Inside the shielding box, three planes of detectors were aligned in a mini-crate by butting the detectors to the optical mirror; the crate was held on a translation-rotation stage. The translation-rotation stage could move the crate in the $x$-direction, rotate in the $\phi$ angles around the $y$-axis, the $\theta$ angles around the (rotated) $x$-axis. Three planes of the detectors were separated by $8 \mathrm{~mm}$ each to minimize the effect of multiple Coulomb scattering. For 4 $\mathrm{GeV} / \mathrm{c}$ pions, the r.m.s. deviation of the middle plane out of the straight line through the $1 \mathrm{st}$ and 3 rd planes was expected to be $0.3 \mu \mathrm{m}$.

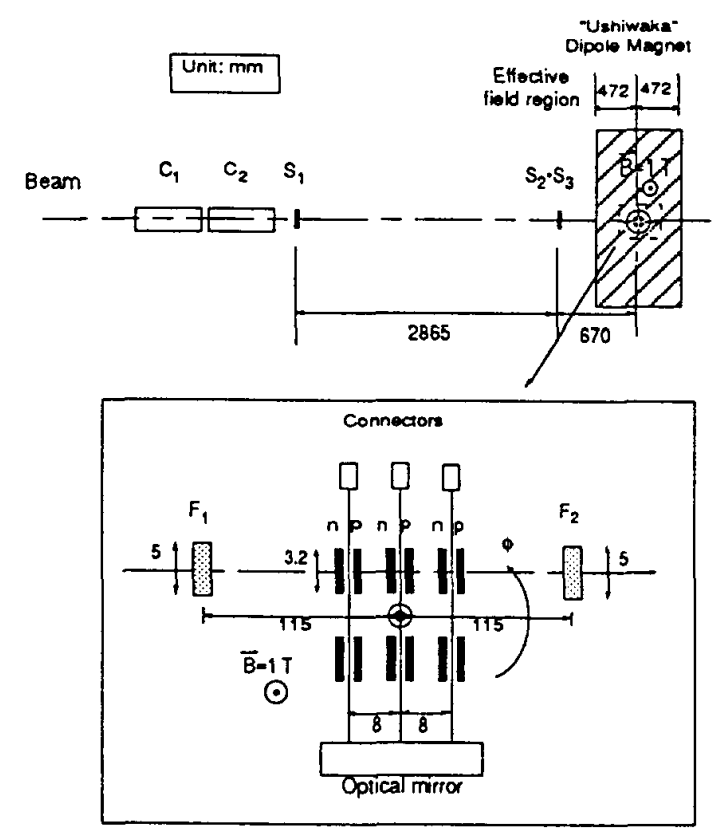

Fig. I Beamline sctup of the T280 experiment used to evaluate the performance of the first prototype SDC double-sided silicon microstrip sensor.

\section{Detectors}

Three identical detectors were fabricated for the beam test. One detector was made of one DSSS on a $12 \mathrm{~cm} \times 12 \mathrm{~cm}$ multi-layer PC board (Fig. 2). The basic building block of the readout front-end electronics was a pair of analog and digital LSI's. This scheme, an analog LSI being realized with bipolar technology and a digital LSI with CMOS technology, has been identified as being the best-suited for a fast, lownoise, and low-power consumption readout for the SDC detector [4]. In this detector board, there were two sets in each side of the board. The analog chips were TEKZ bipolar chips [5], having input/output of 64 channels and unipolar (Poisson) shaping with a time constant of $\sim 30 \mathrm{~ns}$. The digital chips were CMOS SRAM buffer chips [6].

A block diagram of the sensor and the front-end electronics is shown in Fig. 3. A DSSS was reversely biased at $100 \mathrm{~V}$ with a single DC power supply. The reference point which was connected to the front-end amplifier ground was made by dividing the potential with two $40 \mathrm{M} \Omega$ resistors. This was to supply a symmetric potential difference to the AC coupling capacitors of the p- and n-sides of the DSSS. Signals were amplified, shaped, and discriminated (time over the threshold) to on-off levels with the analog chips. The discrimination threshold voltage $\left(V_{t}\right)$ was controlled separately for the p-side and the n-sides (but not separately for the three detectors) and was set at $1.2 \mathrm{fC}$. The on-off levels were transmitted to the digital chips, in which the levels were sampled and pipelined through the first level buffer of 64-bit depth at a $10 \mathrm{MHz}$ clock continuously. With a trigger accept, which was asynchronous to the phase of the clock, a bunch of bits ( 3 in this experiment) was transferred to the level- 2 buffer and then transferred to the output register for readout with the $10 \mathrm{MHz}$ clock.

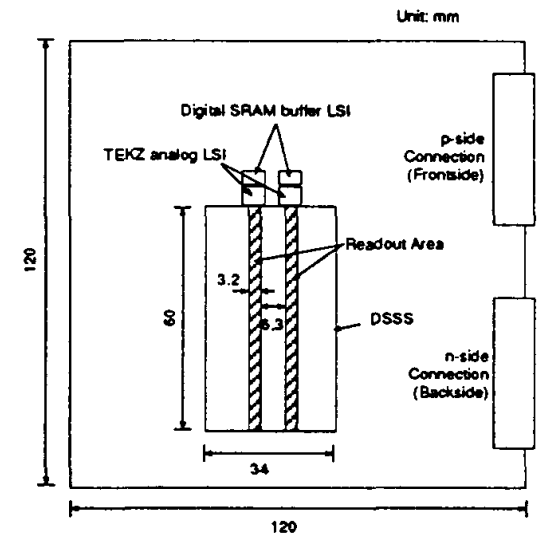

Fig. 2 Detector board layout used for the experiment. One DSSS was read out with two sets of 64 channel analog and digital LSI's per side. The two sets covered two separated $3.2 \mathrm{~mm}$ wide areas of the DSSS.

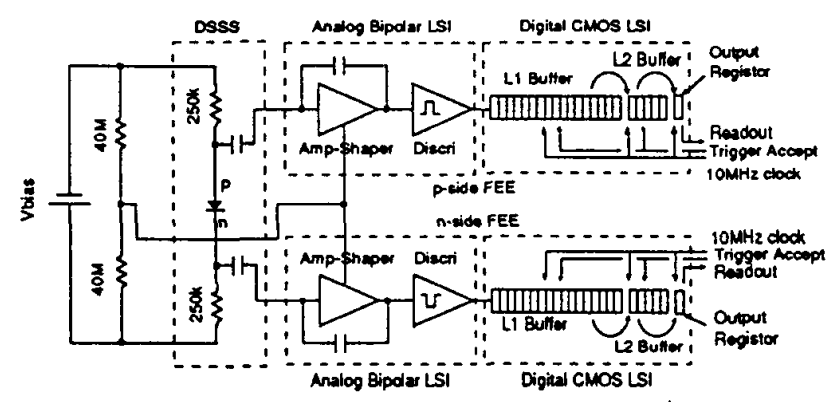

Fig. 3 Schematic block diagram of the DSSS and the front-end electronics. A $10 \mathrm{MHz}$ clock was supplied continuously. regardless of trigger accepts. 


\section{Data Acquisition Flow}

Fig. 4 shows the data-acquisition (DAQ) flow. The main feature of the DAQ was the use of the VME-UNIX system (UNIDAQ), which had been developed for experiments at the SSC [7]. A UNIX workstation (DEC station 5000/125) controlled the VME modules through the DEC Turbo-channel VME adapter in the workstation and the DEC PMABVT6000-AA VME module in the VME crate. CAMAC modules were communicated via the VME.

A total coincidence of $S_{1} \cdot S_{2} \cdot S_{3} \cdot F_{1} \cdot F_{2}$ initiated the DAQ flow: a VME digital readout sequencer (DRS) module [8] to transfer the data from the front-end digital LSI's to a memory in the DRS and a CAMAC input register for the work station to process the data in the CAMAC and the VME modules. The DRS module received a $10 \mathrm{MHz}$ clock, threshold voltages $\left(V_{t}\right)$ and calibration signals to route to the detectors. The phase difference (in timc) of a trigger and the clock was measured with a CAMAC TDC.

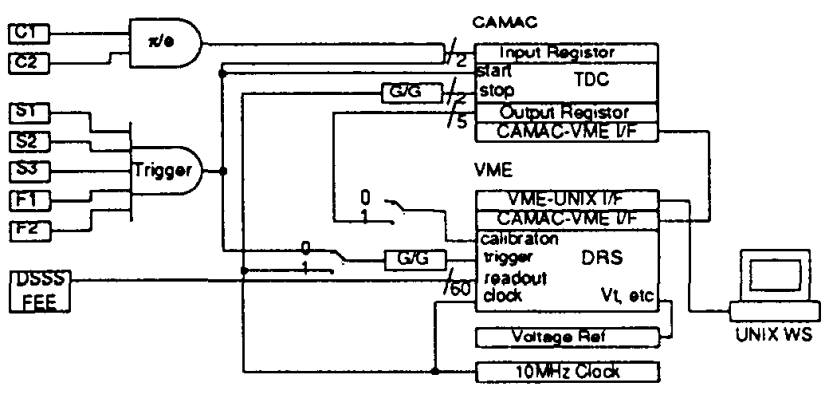

Switch 0: data 1 :calibration

Fig. 4 Data-acquisition flow diagram. The main feature of the DAQ was the use of a VME-UNIX system (UNIDAQ).

\section{Data Taking}

Negatively charged pions of $4 \mathrm{GeV} / \mathrm{c}$ illuminated the center of the readout arca of the connector-side chip set in an area of $5 \times 10 \mathrm{~mm}^{2}$ (The r.m.s size of the beam was $-1 \mathrm{~cm}$ ). The repetition cycle of the beam spill from the $12 \mathrm{GeV} / \mathrm{c}$ PS was $2 \mathrm{sec}$ out of an acceleration-extraction cycle of $4 \mathrm{sec}$. A typical number of triggers was $\sim 1,000$ per spill. The data-acquisition speed was $\sim 200$ events per spill.

The bias voltage was kepl at $100 \mathrm{~V}:-50 \mathrm{~V}$ to the $\mathrm{p}$-side and $+50 \mathrm{~V}$ to the $\mathrm{n}$-side relative to the amplifier ground. Data were collected in the null magnetic field $(B=0 \mathrm{~T})$ for $\phi$-rotations (Set\#1) and for $\theta$-rotations (Set\#2,3,4), and in the 0.9937 Tesla magnetic field $(B=1 \mathrm{~T})$ (Set\#5). The threshold was kept at 1.2fC. At the normal incidence (Sel\#6), the thresholds were varied from 0 to $6 \mathrm{fC}$ at a step of $0.5 \mathrm{fC}$. The conditions of the data set are summarized in Table II. The typical data quantity was $100 \mathrm{k}$ events per data point.
TABLE II.

SUMMARY OF DATA TAKEN FOR THE T280 BEAM TEST

\begin{tabular}{lll} 
Set & Variable & Fixed \\
\hline$\# 1$ & $\phi=0^{\circ}, \pm 3^{\circ}, \pm 6^{\circ}, \pm 9^{\circ}$ & $\theta=0^{\circ}, V_{t}=1.2 \mathrm{fC}, B=0 \mathrm{~T}$ \\
$\# 2,3,4 \quad \theta=0^{\circ}, \pm 18^{\circ}, \pm 36^{\circ}, \pm 54^{\circ}$ & $\phi=0^{\circ}, \pm 9^{\circ}$, \\
& & $V_{t}=1.2 \mathrm{fC}, B=0 \mathrm{~T}$ \\
$\# 5$ & $\phi=0^{\circ}, \pm 1.5^{\circ}, \pm 3^{\circ}, \pm 4.5^{\circ}, \pm 6^{\circ}, \pm 7.5^{\circ}, \pm 9^{\circ}$ \\
& & $\theta=0^{\circ}, V_{t}=1.2 \mathrm{fC}, B=1 \mathrm{~T}$ \\
$\# 6$ & $V_{t}=0 \sim 6 \mathrm{fC}(0.5 \mathrm{fC}$ step $)$ & $\phi=\theta=0^{\circ}, B=0 \mathrm{~T}$ \\
\hline
\end{tabular}

\section{RESULTS}

\section{Determination of Global Alignment}

The alignment of the three detectors was obtained by using data set \#1. Straight tracks were fitted to the clear events with unique hits in 6 measurement planes ( $p$ - and $n$-side $\times$ three detectors), assuming an incident angle of $-\phi$. Six alignment parameters, $x$ - and $y$-displacements and a rotation around $z$ axis of the 2 nd and the 3 rd detectors relative to the 1 st detector $\left((\Delta x, \Delta y, \Psi)_{2}\right.$ and $\left.(\Delta x, \Delta y, \Psi)_{3}\right)$ were obtained by minimizing the distances of the fitted tracks to the axial and the stereo strips of the three planes at $\phi=0^{\circ}$. By fixing these six parameters, the relative $z$-displacements from the nominal $8 \mathrm{~mm}$ of the $2 \mathrm{nd}$ and $3 \mathrm{rd}$ detectors $\left(\Delta z_{2}\right.$ and $\left.\Delta z_{3}\right)$ were obtained, while again minimizing the distances using the angled tracks at $\phi \neq 0^{\circ}$. These obtained 8 global alignment parameters are summarized in Table III.

TABLE III

GLOBAL ALIGNMENT PARAMETERS

\begin{tabular}{ccccl}
\hline Detector\# & $\Delta x(\mu \mathrm{m})$ & $\Delta y(\mu \mathrm{m})$ & $\Delta z(\mu \mathrm{m})$ & $\Psi(\mathrm{mrad})$ \\
\hline 1 & 0. & 0. & 0. & 0. \\
2 & $-350.8 \pm 0.4$ & $-60 \pm 18$ & $-506 \pm 8$ & $-0.53 \pm 0.05$ \\
3 & $-134.9 \pm 0.6$ & $-31 \pm 18$ & $104 \pm 8$ & $-0.25 \pm 0.08$ \\
\hline
\end{tabular}

In finding the hit position, a cluster was defined for the hitstrips adjacent to each other. For a cluster with $n$ hit-strips, the hit position ( $x$ ) was defined using the geometrical mean position of the $n$ strips. Accordingly, the spatial-quantization unit was $25 \mu \mathrm{m}$ in a measurement plane (i.e. p- or n-side).

In the following analysis, three general criteria for the hits were imposed: Clock Timing Cut, Clear Event Cut, and Clear Track Cut.

(1) Clock Timing Cut: Since the clock phase was asynchronous to the trigger, the leading edge of the clock occurred continuously relative to the particle passage. Since the pulse peaking time was $-30 \mathrm{~ns}$, only those events were accepted in a $40 \mathrm{~ns}$ interval where the signal peaks were estimated to fall at about the first $1 / 3$ point.

(2) Clear Event Cut: Because there were dead channels and edges of the readout area, only those events were accepted 
which did not have a hit or an interpolated hit position (defined below) adjacent to the dead channels or to the edge channels.

(3) Clear Track Cut: A straight track was calculated by connecting the cross-point of the p-and n-side hits of the 1 st detector and that of the 3 rd detector. Only those tracks were accepted to have an angular deviation within $\pm 1^{\circ}$ from the nominal values.

In a magnetic field of $1 \mathrm{~T}$, incident charged particles were deflected before arriving at the silicon detectors. This deflection was obtained to be $-2.09 \pm 0.002^{\circ}$ in situ. The $\phi$ angles of the 1T magnetic field were corrected by this amount in the following figures.

\section{Efficiency}

The efficiencics of the $p$ - and the $n$-side of the 2 nd detector were evaluated by counting whether there was a hit in the side. A hit was searched within $\pm 110 \mu \mathrm{m}$ from the interpolated position of the calculated tracks. No obvious inefficiency was observed in the 64 channels of the p-and the n-sides in the three detectors, other than dead channels.

The efficiencies of the $p$ - and the n-side of the 2 nd detector are plotted as a function of $\phi$ angles (Fig. 5). Fig. 5(a) is for $B=0 \mathrm{~T}$ and Fig. $5(\mathrm{~b})$ for $B=1 \mathrm{~T}$. There is an undulation in the data which might have been caused by the loss of signals (see in the discussion in the multi-strip hit fraction); in general, however, the efficiencies wcre as high as $98-99 \%$, regardless of the $\mathrm{p}$ - and the $n$-sides and cxistence of the magnetic field.

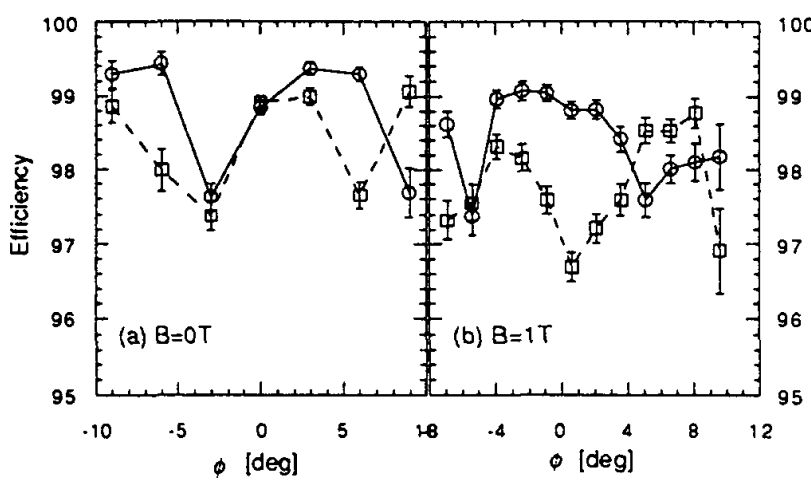

Fig. 5 Efficiency of the p-side (circle/solid line) and n-side (square/dashed line) of the lst prototype SDC double-sided silicon microstrip sensor: (a) no magnetic field, (b) a $1 \mathrm{~T}$ magnetic field.

\section{Position Resolution}

The spatial position resolutions of the $\mathrm{p}$ - and $n$-sides of the 2nd detcctor were estimated using the residuals of the hits in the $\mathrm{p}$ - or n-side to the interpolated position of the calculated track using the hits in the 1 st and the 3 rd detectors, effectively defined by $\delta=\left(x_{1}+x_{3}\right) / 2-x_{2}$. Here, $x$ is the distance normal to the strip. A typical residual distribution is given in Fig. 6, which shows a quantization effect. This quantization of unit of $12.5 \mu \mathrm{m}$ was caused by the quantization of measurements in a plane having a unit of $25 \mu \mathrm{m}$. In order to obtain the resolution, a Gaussian was fitted to the re-binned distribution with a bin size of $12.5 \mu \mathrm{m}$ and the center of the bin at the peak position of the quantization to dismiss the effect.

The formula used to give a single-side resolution was $\sigma_{s}=0.895 \times \sigma_{\delta}$, instead of $\sigma_{s}=\sqrt{2 / 3} \sigma_{\delta}$, by taking into account the $10 \mathrm{mrad}$ stereo angle and the use of two measurement points in defining $x_{1}$ and $x_{3}$. The position resolutions varied as a function of the $\phi$ angles and were $12.5 \mu \mathrm{m}$, where the multi-strip fraction was $30-40 \%$ (Fig. 7).

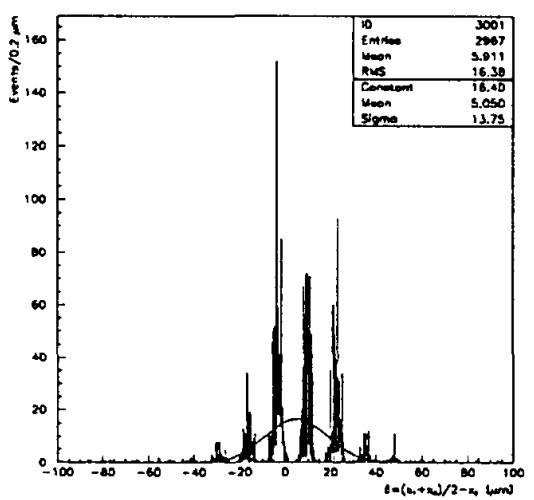

Fig. 6 Typical residual distribution: p-side, $\phi=+8.1^{\circ}, B=1 \mathrm{~T}$.

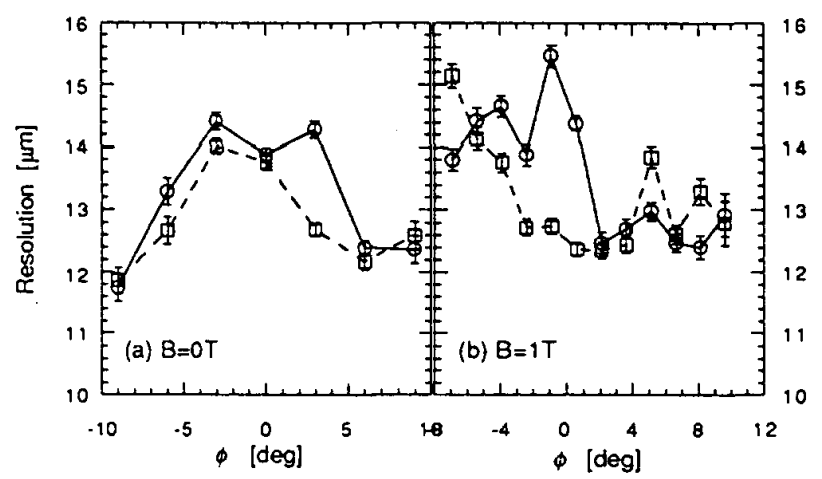

Fig. 7 Position resolutions of the p-side (circles/solid line) and $n$ side (squares/dashed line) as a function of the $\phi$ angles: (a) $B=0 \mathrm{~T}$, (b) $B=1 \mathrm{~T}$

\section{Multi-strip Hit Fraction}

The swath of generated electron-hole pairs along their passages of charged particles in $300 \mu \mathrm{m}$ silicon spreads over more than single strip, depending on the incident angles or the existence of a magnetic field. A signal simulation shows that the position resolution will be improved by more than the pitch $/ \sqrt{12}$ due to the separation of single-strip-hit and doublestrip-hit regions; there is an angle in which electrons and holes are collected over equal sized spans in the p- and n-sides [9]. The Hall mobilities of the electrons and holes must be known to determine this tilt angle.

The multiple-strip hit fraction distributions show clear angular and magnetic field dependencies (Fig. 8). The $n$-side fraction was higher than that of the p-side, possibly due to the 
larger diffusion of electrons and greater electronic noise in the $\mathrm{n}$-side. There is a clear correlation between the fraction and position resolution; the best position resolution was obtained when the fraction was $30-40 \%$. There was, however, a slight anti-corrclation between the fraction and efficiency; the threshold of $1.2 \mathrm{CC}$ might have been a bit too high.

The $\phi$-angles where the fractions are minimum corresponds to the Hall angles of the carriers. The curves in Fig. 8 were fitted lines to a hypcrbolic function, $y=c \sqrt{1+(x-a)^{2} / b}$. After calibrating the $\phi$-angle readout-offsets using the $B=0 \mathrm{~T}$ data, the Hall angles $\left(\theta_{11}\right)$ were obtained to be $-1.85 \pm 0.17^{\circ}$ (holes: pside) and $+7.87 \pm 0.24^{\circ}$ (electrons: $n$-side), which correspond to the Hall mobilities $\left(\mu_{H}\right)$ of $325 \pm 30$ (holes) and $1391 \pm 43$ $\mathrm{cm}^{2} / \mathrm{Vs}$ (electrons) using the relation $\tan \theta_{H}=10^{-8} \mu_{H} B$. The dopant concentration of the silicon was estimated to be $(1.02 \pm 0.15) \times 10^{12} \mathrm{~cm}^{-3}$ from a measurement of the depletion voltage.

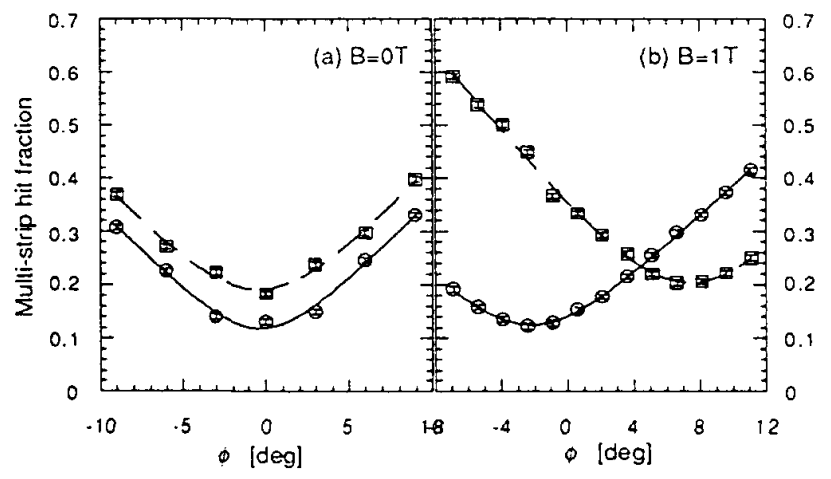

Fig. 8 Multi-strip hit fraction as a function of the $\phi$-angles: (a) $B=0$ T, (b) $B=1 \mathrm{~T}$; p-side (circle/solid line), $\mathrm{n}$-side (square/dashed line). The curves are fitted lines to a hyperbolic function.

$$
y=c \sqrt{1+(x-a)^{2} / b} \text {. }
$$

\section{Signal-to-Noise Ratio}

Using data set \#6 (threshold variation) the efficiencies of the 2 nd detector were obtained as a function of the threshold voltage. By fitting an error function to the variation, the gain of the amplifier was determined by regarding the $50 \%$ point as being the most probable energy deposition of $4 \mathrm{fC}$ in $300 \mu \mathrm{m}$ silicon. The gain was slightly more than $100 \mathrm{mV} / \mathrm{fC}$ for both the $\mathrm{p}$ - and $\mathrm{n}$-sides. The electronic noise was determined from pulsar calibration runs, and was found to be 22 and $25 \mathrm{mV}$ for the $\mathrm{p}$ - and $\mathrm{n}$-sides, respectively. The signal/noise ratios were 18 and 16 , respectivcly.

\section{CONCLUSION}

The first protolype of a radiation-hard double-sided silicon microstrip sensor for the SDC experiment at the SSC was successfully beam tested using $4 \mathrm{GeV} / \mathrm{c}$ pions at KEK. The front-end readout scheme used in the tcst was conceptually the same as the proposed scheme: bipolar analog LSI's and CMOS digital LSI's; binary (on/off) readout; and continuous clocking.
TEKZ analog LSI's ( $\tau \sim 30 \mathrm{~ns}$ ) and CMOS SRAM digital LSI's were used for the readout electronics, and the digital chips were clocked at $10 \mathrm{MHz}$ asynchronously with the beam triggers. The front-end electronics were interfaced with a digital readout sequencer (DRS) to a VME-UNIX data-acquisition system (UNIDAQ). Data were taken in the null and $1 \mathrm{~T}$ magnetic fields and by rotating the three planes of the detectors in $\phi$ and $\theta$ directions.

The efficiencies and position resolutions of the $p$ - and $n$ sides of the middle detector were evaluated as a function of the $\phi$-rotation angles by using the 1 st and 3 rd detectors as the defining anchors. The efficiencies were found to be $98 \sim 99 \%$, depending on the $\phi$-angle and the existence of a magnetic field. The position resolutions were as good as $12.5 \mu \mathrm{m}$, where the multi-strip hit fraction was $30-40 \%$. There was no essential difference in the performance of the $\mathrm{p}$ - and the $\mathrm{n}$-sides. The multi-strip hit fraction showed a clear rotation and a magneticfield dependence. From the angles where the fractions were minimum in the $1 \mathrm{~T}$ magnetic field, the Hall mobilities of the electrons and holes were obtained to be $1391 \pm 43$ (electrons) and $325 \pm 30$ (holes) $\mathrm{cm}^{2} / \mathrm{Vs}$, respectively.

\section{ACKNOWLEDGMENTS}

This work was supported in part by the Japan-US cooperation in the field of high-energy physics, by a Grant-inAid for Joint Research in the International Scientific Research Program of Ministry of Education, Science and Culture, of Japan, and by the US Department of Energy. The authors would like to thank the support of the staff of the PS section of KEK.

\section{REFERENCES}

[1] T. Ohsugi et al., Double-sided Microstrip Sensor for the Barrel of the SDC Silicon Tracker, in the Int. Symp. Dev. Appl. of Semiconductor Tracking Detectors (Hiroshima STD Symposium), May 22-24, 1993, Hiroshima, the proceedings to be published in Nucl. Instr. Meth. Sec. A; T. Ohsugi, et al., Prototype Double Sided Silicon Sensor (DSSS) for SDC Detector, IEEE Nucl. Scie. Symp. at Orlando, Oct. 25-30, 1992. The microstrip sensors were fabricated by Hamamatsu Photonics, Co. Ltd, Hamamatsu 430, Japan

[2] A. Weinsten et al., Silicon Tracking Conceptual Design Report, SCIPP 92/04, University of California, March 1992

[3] Y. Unno, The SDC Silicon Tracking System, in the Hiroshima STD Symposium

[4] Ref. 2; H. Spieler and D. Dorfan in the Hiroshima STD Symposium.

[5] E. Barberis, et al., IEEE Trans. Nucl. Scie. 40(1993)740

[6] J. DeWitt, Nucl. Instr. Meth. A288(1990)209

[7] A. Fry and M. Nomachi, UNIDAQ: a portable data-acquisition system for SSC detector R\&D, 8th Real-time Computer Applications in Nuclear, Particle, and Plasma Physics (RT93). June 8.11, 1993, Vancouver, Canada

[8] B. Hubbard et al., A Digital Readout Sequencer (DRS), SCIPP 93/43, Univ. of California, Santa Cruz

[9] J. Leslie, A. Seiden, Y. Unno, SCIPP 92/20, Univ. of California, Santa Cruz; IEEE Trans. Nucl. Scie., 40(1993)557 
